Revista Energia na Agricultura

ISSN 1808-8759

\title{
AVALIAÇÃO DO PROCESSO DE HIDRÓliSE ENZIMÁTICA PARA OBTENÇÃO DE AÇÚ- CARES A PARTIR DE GENGIBRE (Zingiber officinale) ${ }^{1}$
}

LÍVIA MARIA TORRES ${ }^{2} \&$ MAGALI LEONEL ${ }^{3}$

RESUMO: O gengibre é uma tuberosa amilácea bastante valorizada pelos seus componentes químicos. Na produção de alguns tipos de bebidas, tem sido usado o extrato de gengibre; contudo, frente ao seu elevado conteúdo de amido, uma possibilidade de incremento de renda para o setor agroindustrial desta tuberosa seria a hidrólise de rizomas desclassificados para exportação e o uso dos hidrolisados para a obtenção de bebidas fermentadas. Neste trabalho, objetivou-se avaliar o processo de obtenção de açúcares a partir de rizomas de gengibre, sendo testadas duas enzimas alfa-amilases na etapa de liquefação (Liquozyme Supra (T1) e Termamyl 2X (T2)), bem como o efeito do tempo de ação da amiloglucosidase (AMG 300L). Os hidrolisados obtidos foram analisados em cromatografia líquida (CLAE) e realizou-se o balanço de massa dos processos. Os resultados obtidos mostraram maior hidrólise do amido no tratamento que utilizou a Liquozyme Supra. O tempo de ação de 18 horas da AMG 300L proporcionou um hidrolisado onde $98 \%$ de seus componentes químicos era glicose.

Palavras-chave: Amido, amilases, rendimento.

\footnotetext{
${ }^{1}$ Parte da dissertação de mestrado do $1^{\circ}$ autor intitulada: "Caracterização dos parâmetros técnicos do processo de fabricação de aguardente a partir de gengibre".

${ }^{2}$ Aluna do Curso de PG Energia na Agricultura - FCA/UNESP - Botucatu/SP - Brasil.

${ }^{3}$ Orientadora e Pesquisadora do Centro de Raízes e Amidos Tropicais- CERAT/UNESP- Botucatu/SP - Brasil.
} 


\section{EVALUATION OF ENZYMATIC HYDROLISIS PROCESS TO OBTATION OF SUGARS FROM GINGER (ZINGIBER OFFICINALE)}

SUMMARY: Ginger is a starchy tubers prized for their chemical components. In the production of any kinds of beverages has been added to the extract of ginger. However, in view of the high starch content, a possibility of further development of the agribusiness sector this would be the hydrolysis tuberous rhizomes disqualified for export in order to obtain hydrolysates that would be used in the preparation of fermented beverages. This work aimed to evaluate the production of sugar from rhizomes of ginger. Two $\alpha$-amylase enzymes were tested in the stage of liquefaction (Liquozyme Supra (T1) and Termamyl $2 X$ (T2)), as well as the effect of time of action of amyloglucosidase (AMG 300L). The hydrolysates were analyzed in liquid chromatography (HPLC) and was also carried out the mass balance of the processes. The results showed higher hydrolysis of starch in the treatment that used Liquozyme Supra in liquefaction. The action time of 18 hours of AMG 300L hydrolyzate which gave an $98 \%$ of its chemical components was glucose.

Keywords: Starch. amylases, yield

\section{INTRODUÇÃO}

O gengibre (Zingiber officinale) é uma planta pertencente à família Zingiberaceae, que produz um rizoma muito valorizado por seus diversos usos tanto na culinária, como na medicina popular, além da utilização como planta ornamental e nas indústrias de perfumes e bebidas (BLANCO, 1997).

A produção mundial de gengibre em 2007 foi de 1,3 milhões de toneladas, sendo a Índia o maior produtor mundial (420 mil toneladas), seguida pela China (285 mil toneladas), Indonésia (177 mil toneladas), Nepal (158,9 mil toneladas) e Nigéria (138 mil toneladas) (Food and Agriculture OrganizationFAO, 2008).

O Brasil se inclui entre os pequenos produtores de gengibre, cuja produção é orientada para exportação. A quantidade de rizomas destinada à exportação representa 70 a $80 \%$ da colheita, havendo uma perda de 20 a $30 \%$ por ocasião do beneficiamento, para os rizomas que não atingem qualidade para exportação (TAVEIRA-MAGALHÃES et al., 1997). 
Os rizomas de gengibre do tipo caipira apresentam em média 84,37\% de umidade, 1,17\% de proteína, 0,86\% de lipídeos, 0,93\% de fibras, 0,96\% de cinzas, 11,42\% de amido e 0,34\% de açúcares (LEONEL et al., 2005).

$\mathrm{O}$ amido constitui o mais abundante carboidrato de reserva das plantas superiores, presentes nos tecidos sob forma de grânulos intracelulares. Os polímeros formadores do grânulo de amido são a amilose, de cadeia linear de $(1 \rightarrow 4)$ - $\alpha$-D-glucose e a amilopectina, molécula ramificada, onde cadeias de $(1 \rightarrow 4)$ - $\alpha$ D-glucana são conectadas por ligações $\alpha(1 \rightarrow 6)$ (BILIADERIS, 1991).

O tamanho e a forma dos grânulos de amido são característicos da fonte botânica, sendo que os grânulos de amido de gengibre apresentam formas circular e ovalada e distribuição de tamanho homogênea, com predomínio de diâmetro maior médio na faixa de 15 a 20 m (LEONEL, 2007).

$\mathrm{Na}$ presença de água e calor os grânulos de amido expandem-se embebendo água. Com o aquecimento, a temperatura de gelatinização é atingida e uma pasta é formada. As propriedades do amido de gengibre mostram elevada temperatura de pasta $\left(91,1^{\circ} \mathrm{C}\right)$, baixo pico de viscosidade (78 RVU), baixa quebra de viscosidade (próxima a zero) indicando a presença de forças de ligações homogêneas e a um elevado grau de associação entre os componentes deste amido, mantendo a estrutura granular (PERONI, 2003; VIEIRA, 2004; LEONEL et al., 2005).

A hidrólise do amido pode ser por via ácida ou enzimática. Os produtos de conversão enzimática do amido vão de dextrinas de peso molecular elevado à glicose. Uma das características mais importantes da catálise enzimática é sua especificidade, muito maior do que a da catálise química, quanto à reação e quanto ao substrato.

As amilases são enzimas que atuam hidrolisando cadeias de amido, quebrando as ligações glicosídicas $\alpha-1,4$, enquanto as pululanases e isoamilase atuam nas ligações $\alpha-1-6$ (EL-DASH et al., 1982; OBEL, 2001).

A ação sinérgica da alfa-amilase e da amiloglucosidase no processo de hidrólise vem sendo estudada por diversos pesquisadores em amidos de diferentes origens. Monna et al. (1989), estudaram a eficiência do uso destas duas enzimas em grânulos de amido de arroz, sagu e batata e concluíram que esta combinação apresenta bons rendimentos na conversão do amido à glicose em todos os substratos. Leonel e Cereda (1999) estudaram enzimas complementares no processo de hidrólise de amido de mandioca, para avaliar a eficiência no uso destes coadjuvantes na obtenção de glicose.

A enzima Liquozyme é uma alfa-amilase otimizada para reduzir o comprimento da cadeia de dextrina e a viscosidade do mosto, antes da sacarificação e tem sido utilizada para a hidrólise de amido de milho e batata (DEVANTIER et al., 2005; SRICHUWONG et al., 2009).

Diante das características químicas do amido de gengibre e da importância do conhecimento do processo de sua hidrólise, visando o seu possível uso nas indústrias de bebidas, objetivou-se neste trabalho 
avaliar o uso de duas diferentes alfa-amilases comerciais, bem como, o tempo de ação da amiloglucosidase para a obtenção de hidrolisado com elevado teor de glicose a partir de rizomas de gengibre.

\section{MATERIAL E MÉTODOS}

Rizomas frescos de gengibre do tipo caipira, produzidos na região de Tapiraí-SP, foram caracterizados quanto aos teores de umidade, açúcares totais e amido (American Association of Cereal ChemistsAACC, 1983; SOMOGY, 1945; ISO, 1987).

No preparo das suspensões de gengibre com $10 \%$ de amido, foram utilizadas a massa de gengibre desintegrado em cevadeira e água. Foram preparadas suspensões de $6 \mathrm{~kg}$ e o processo de hidrólise foi conduzido em reator em aço inox, com temperatura e agitação controladas, e capacidade de 18 litros.

No processo de hidrólise foram realizados dois tratamentos para a comparação da ação de duas $\alpha$ amilases em conjunto com uma amiloglucosidade nas etapas de liquefação e sacarificação das suspensões de gengibre. No tratamento 1, utilizou-se para a liquefação a Liquozyme Supra, e no tratamento 2 a Termamyl 2X. Após a ação das $\alpha$-amilases adicionou-se a amiloglucosidase AMG 300L nos dois tratamentos.

Para a comparação da ação das duas $\alpha$-amilases na liquefação da suspensão foram mantidos como parâmetros fixos do processo a concentração (1,2 kg de enzima/ tonelada de amido) e o tempo de reação ( 2 h). Na primeira hora, o reator foi mantido a $105^{\circ} \mathrm{C}$, com a adição de $1 / 3$ da quantidade de enzima, sob agitação constante e, na segunda hora, a temperatura era reduzida para $95^{\circ} \mathrm{C}$ e adicionados os $2 / 3$ restantes da enzima. Na sacarificação pela amiloglucosidase (AMG 300L) utilizou-se a concentração de 2,25 litros de enzima/tonelada de amido, e temperatura de reação de $60^{\circ} \mathrm{C}$ (Figura 1).

Amostras de cada tratamento foram retiradas e filtradas nos tempos 2, 18, 24, 36, 42, 48, 66 e 72 horas para análises do perfil dos açúcares. O resíduo fibroso foi pesado e caracterizado para os teores de umidade, açúcares totais e amido (AACC, 1983; SOMOGY, 1945; ISO, 1987).

O perfil dos açúcares no hidrolisado de gengibre foi analisado em cromatógrafo líquido (CLAE), marca Varian modelo Pró-Star com duas bombas binárias e índice de refração (detector) com amostrador automático, sendo a coluna de marca BIORAD modelo AMINEX HPX 87P (fase estacionária $\mathrm{Pb}$ ) $300 \mathrm{x}$ $0,25 \mathrm{~mm}$, utilizando como fase móvel água e fluxo da amostra de $0,6 \mathrm{ml} /$ minuto, na temperatura de $80^{\circ} \mathrm{C}$, que permite a quantificação de sacarídeos de $\mathrm{Dp}$ de 1 a 9 . Os perfis em área foram convertidos em concentração $(\mathrm{g} / \mathrm{L})$ a partir de curvas padrões de glicose, frutose, maltose e sacarose. 


\section{SUSPENSÕES DA MATÉRIA-PRIMA}

(pH 5,6)

Adição de 1/3 das enzimas:Termamyl 2X (70 ppm de cálcio) ou Liquozyme Supra $2 \mathrm{X}(5 \mathrm{ppm}$ de cálcio)

Aquecimento e agitação até atingir $105^{\circ} \mathrm{C}$

Manutenção da temperatura e agitação por 1 hora

Redução da temperatura para $95^{\circ} \mathrm{C}$

Adição de 2/3 das enzimas

Manutenção da temperatura e agitação por 1 hora

Resfriamento para $60^{\circ} \mathrm{C}$ e ajuste para $\mathrm{pH} 4,5$

Adição de 2,25 L de AMG / $\mathrm{t}$ amido

Manutenção da temperatura em $60^{\circ} \mathrm{C}$ com agitação por 72 horas

Filtração

HIDROLISADO

Figura 1 - Processo de hidrólise-sacarificação das suspensões de gengibre.

Os rendimentos dos processos de hidrólise enzimática foram calculados através da divisão da soma do total de açúcares obtidos no hidrolisado de gengibre e dos açúcares totais retidos no resíduo, pelo valor teórico de glicose inicial, calculado pela conversão estequiométrica do amido em glicose somada ao teor de açúcar total presentes nos rizomas de gengibre. Já os rendimentos finais dos processos foram calculados pela divisão total de açúcares presentes nos hidrolisados pela quantidade teórica de açúcares, não sendo considerados os açúcares retidos nos resíduos.

A comparação da ação das $\alpha$-amilases no perfil de açúcares do hidrolisado após a liquefação e também dos diferentes tempos de ação da amiloglucosidase foi realizada através de análise de variância e a seguir pela comparação das médias pelo teste de Tukey. O nível de significância adotado foi de 5\%. Para a análise de glicose no hidrolisado durante a ação da AMG 300L, foi realizada a análise de variância da regressão polinomial dos tempos de ação da enzima, verificando as interações entre as regressões e os tratamentos; foram determinadas as equações de regressão e construído o gráfico (CAMPOS, 1984). 


\section{RESULTADOS E DISCUSSÃO}

Os resultados obtidos para o perfil de açúcares no hidrolisado de gengibre após a etapa de liquefação (2h) mostraram ocorrência de diferenças significativas entre os tratamentos enzimáticos (Tabela 1).

No tratamento em que se utilizou a Termamyl 2X (T2) observou-se uma menor porcentagem de dextrinas e glicose e maiores porcentagens dos demais açúcares quando comparado com o tratamento com a Liquozyme Supra (T1).

O teor de glicose no hidrolisado após a ação de amilases é determinado pela dosagem da enzima, condições de processo e eficiência da enzima. Segundo o fabricante das enzimas, a Liquozyme Supra $(0,4 \mathrm{Kg} / \mathrm{t}$ matéria seca, $5 \mathrm{ppm} \mathrm{Ca}, \mathrm{pH}$ 5,5) apresenta uma maior eficiência na produção de dextrose equivalente $(11 \%)$ quando comparada com a Termamyl 120L (0,4Kg/t matéria seca, 70ppm Ca, pH 6,2) após duas horas de ação (9\%) (NOVOZYMES, 2008).

Tabela 1- Comparação das médias da concentração dos açúcares presentes no hidrolisado de gengibre após a ação das duas $\alpha$-amilases.

\begin{tabular}{ccc}
\hline Açúcares (\%) & Liquozyme Supra (T1) & Termamyl 2X (T2) \\
\hline Dextrinas & $77,67 \mathrm{a}$ & $72,39 \mathrm{~b}$ \\
Sacarose & $1,17 \mathrm{~b}$ & $3,50 \mathrm{a}$ \\
Maltotetraose & $1,89 \mathrm{~b}$ & $2,21 \mathrm{a}$ \\
Maltotriose & $4,37 \mathrm{~b}$ & $6,91 \mathrm{a}$ \\
Maltose & $1,70 \mathrm{~b}$ & $2,93 \mathrm{a}$ \\
Glicose & $13,19 \mathrm{a}$ & $12,05 \mathrm{~b}$ \\
\hline
\end{tabular}

* Médias seguidas de mesma letra na linha não diferem entre si a 5\% pelo teste de Tukey.

Os teores de glicose nos hidrolisados de gengibre apresentaram diferenças significativas dentro de cada tempo de ação da amiloglucosidase, evidenciando em todos os tempos, maiores concentrações de glicose nos hidrolisados em que se utilizou a Liquozyme Supra (Tabela 2). 
Tabela 2 - Comparação do teor de glicose (g/L) nos hidrolisados obtidos após a liquefação pelas duas $\alpha$ amilases, em diferentes tempos de ação da AMG 300L.

\begin{tabular}{ccc}
\hline Tempo (h) & Liquozyme Supra (T1) & Termamyl 2X (T2) \\
\hline 18 & $106,37 \mathrm{a}$ & $85,54 \mathrm{~b}$ \\
24 & $106,37 \mathrm{a}$ & $92,31 \mathrm{~b}$ \\
42 & $107,28 \mathrm{a}$ & $92,51 \mathrm{~b}$ \\
48 & $109,11 \mathrm{a}$ & $93,41 \mathrm{~b}$ \\
66 & $110,52 \mathrm{a}$ & $94,53 \mathrm{~b}$ \\
72 & $111,13 \mathrm{a}$ & $95,08 \mathrm{~b}$ \\
\hline
\end{tabular}

* Médias seguidas de letra diferentes na mesma linha diferem entre si ao nível de 5\% pelo teste de Tukey.

A análise do efeito do tempo de ação da amiloglucosidase sobre a porcentagem de glicose nos hidrolisados mostrou aumento linear deste açúcar no tratamento com Liquozyme Supra. No tratamento com Termamyl $2 \mathrm{X}$ o teor de glicose aumentou significativamente das $18 \mathrm{~h}$ para as $24 \mathrm{~h}$ de ação da amiloglucosidase (Figura 2).

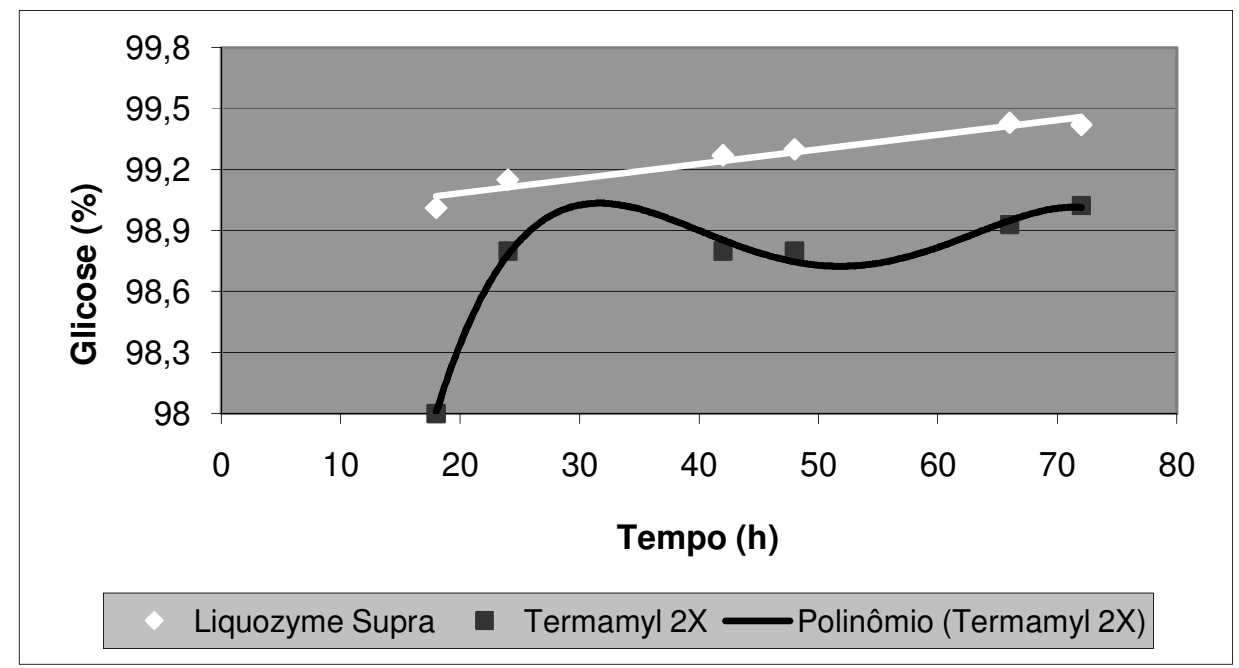

Liquozyme Supra: $Y=0,0072 x+98,938\left(R^{2}=0,9432\right)$

Termamyl 2X: $Y=87,59+1,0439 x-0,0339 x^{2}+0,0005 x^{3}-2 E-06 x^{4}\left(R^{2}=0,9888\right)$

Figura 2 - Regressão linear para o tratamento com Liquozyme Supra e regressão de $4^{\circ}$ grau para o tratamento do Termamyl $2 \mathrm{X}$ das 18 às 72 horas de ação da AMG300L. 
Através da análise dos resultados obtidos na caracterização dos resíduos finais, bem como dos hidrolisados, estabeleceu-se o balanço de massa dos diferentes tratamentos (Figura 3). Os rizomas de gengibre apresentaram 83,09\% de umidade, $11,4 \%$ de amido e 0,8\% de açúcares totais. Portanto, os processos de hidrólise iniciaram para os dois tratamentos com 889,5g de matéria seca, 600g amido $(67,41 \%)$ e $42,07 \mathrm{~g}$ de açúcares totais $(4,73 \%)$, o que levaria a um rendimento teórico de 666,66 g de glicose do amido ou 708,74g de açucares totais. Após a hidrólise, observou-se que 14,33\% do amido inicial e, 19,7\% do total de açúcares obtidos, ficaram retidos no resíduo fibroso do tratamento em que se utilizou a Liquozyme Supra (T1) e o rendimento de hidrólise foi de 75,55\%. Já para o tratamento com a Termamyl 2X (T2), ficaram retidos no resíduo fibroso $11,21 \%$ do amido inicial e, 22,86\% dos açúcares obtidos, com um rendimento de hidrólise de $71,10 \%$. Os rendimentos finais dos processos de obtenção de açúcares a partir de rizomas de gengibre foram de 60,7\% para Liquozyme Supra e 54,85\% para a Termamyl 2X.

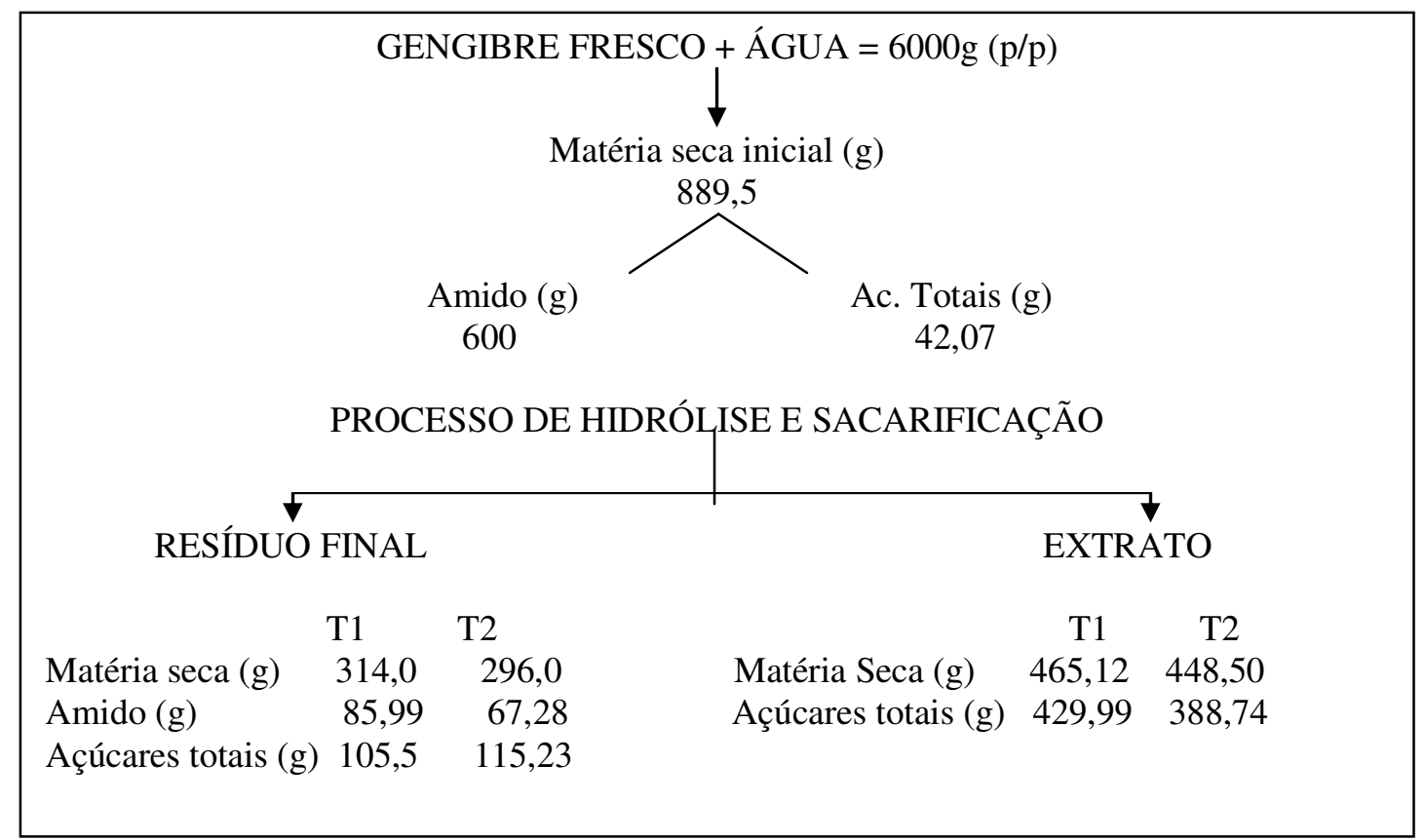

Figura 3 - Balanço de massa do processo de hidrólise-sacarificação do rizoma de gengibre nos diferentes tratamentos. 
O maior rendimento de hidrólise com a Liquozyme Supra na etapa de liquefação pode ser devido à redução da viscosidade, facilitando a recuperação dos açúcares na etapa de filtração. (DEVANTIER et al., 2005; SRICHUWONG et al., 2009).

Leonel e Cereda (1998) avaliando o rendimento de hidrólise enzimática do resíduo fibroso da industrialização da mandioca observaram que $86,31 \%$ do amido foi hidrolisado quando se utilizou pectinase para a redução da viscosidade na etapa de liquefação, resultado semelhante ao obtido nos dois tratamentos $(85,67 \%$ no $\mathrm{T} 1$ e $88,79 \%$ no $\mathrm{T} 2)$.

A porcentagem de amido hidrolisado nos dois tratamentos foi superior a relatada em estudo da ação de enzimas complementares Termamyl 120L e AMG 300L na hidrólise enzimática de farelo de mandioca, no qual se obteve $63,42 \%$ do total de amido hidrolisado no tratamento que utilizou apenas estas duas enzimas (LEONEL;CEREDA, 1999).

\section{CONCLUSÕES}

1) O uso da Liquozyme Supra na etapa de liquefação da suspensão de gengibre promoveu melhor rendimento de processo na hidrólise de rizomas de gengibre;

2) Independente da $\alpha$-amilase utilizada na liquefação obtêve-se hidrolisado com $98 \%$ de glicose após 18 horas de ação da amiloglucosidase (AMG 300L).

\section{AGRADECIMENTO}

À Novo Nordisk Bioindustrial do Brasil - LTDA pela doação das enzimas utilizadas neste trabalho.

\section{REFERÊNCIAS}

AMERICAN ASSOCIATION OF CEREAL CHEMISTS. Approved methods of the American Association of Cereal Chemists. 7th ed. rev. St. Paul, 1983. 
BILIADERIS, C. G. The estructure and interactions of starch with food. Canadian Journal of Physiology and Pharmacology, Ottawa, v. 69, p. 60-78, 1991.

BLANCO, M. C. G. Gengibre (Zingiber officinale). In: COORDENADORIA DE ASSITÊNCIA TÉCNICA INTEGRAL. Manual técnico das culturas. v.2: Olerícolas, Medicinais e Ornamentais. 2ed. Campinas, 1997.v. 2, p. 179-182.

CAMPOS, H. Estatística aplicada à experimentação com cana-de açúcar. Piracicaba: FEALQ, 1984. $292 \mathrm{p}$.

DEVANTIER, R.; PEDERSEN, S.; OLSSON, L. Characterization of very high gravity ethanol fermentation of corn mash. Effect of glucoamylase dosage, pre-saccharification and yeast strain. Applied Microbiology Biotechnology, Germany, v. 68, p. 622-629, 2005.

EL-DASH, A. A.; CAMARGO, C. O; DIAZ, N. M. Fundamentos da tecnologia de panificação. São Paulo: Governo do Estado de São Paulo, 1982. 349 p.

FAO. Base de dados. 2008. Disponível em: <http://www.fao.org>. Acesso em 3 set. 2008.

INTERNATIONAL ASSOCIATION FOR STANDARDIZATION. ISO 6647/1987: rice- determination of amylose content, 1987.

LEONEL, M.; CEREDA, M. P. Avaliação da celulase e pectinase como enzimas complementares no processo de hidrólise-sacarificação do farelo de mandioca para produção de etanol. Ciência e Tecnologia de Alimentos, Campinas, v. 19, p. 113 - 117, 1999.

LEONEL, M.; SARMENTO, S. B. S.; FERRARI, T. B. Aproveitamento do gengibre (Zingiber officinale) de qualidade inferior como matéria-prima amilácea. Revista Raízes e Amidos Tropicais, Botucatu, v. 1, p. 9-18, 2005.

LEONEL, M. Análise da forma e tamanho de grânulos de amido de diferentes fontes botânicas. Ciência e Tecnologia de Alimentos, Campinas, v. 27, n. 3, p. 579-588, 2007.

MONNA, M. et al. Starch Stärke , v. 41, p. 382-385, 1989. 
NOVOZYMES. Starch application sheet: eficient liquefaction of starch. Disponível em:<http:// www.novozymes.com>. Acesso em: 14 set. 2008.

OBEL, L. B. Putting enzymes to work in bakery applications. Cereal Foods World. v. 46, n. 9, p. 396399, 2001.

PERONI, F. H. G. Características estruturais e físico-químicas de amidos obtidos de diferentes fontes botânicas. 2003. 107 p. Dissertação (Mestrado em Ciência e Tecnologia de Alimentos)-Instituto de Biociências, Letras e Ciências Exatas, Universidade Estadual Paulista, São José do Rio Preto, 2003.

SOMOGY, M. Determination of blood sugar. Journal Biological Chemistry, Baltimore, n.160, p. 69 $73,1945$.

SRICHUWONG, S et al. Simultaneous saccharification and fermentation (SSF) of very high gravity (VHG) potato mash for the production of ethanol. Biomass and Bioenergy, v. 33, p. 890-898, 2009.

TAVEIRA-MAGALHÃES, M. et al. Gengibre (Zingiber officinale Roscoe) brasileiro: aspectos gerais, óleo essencial e oleoresina. Parte1- aspectos gerais, óleo essencial. Ciência e Tecnologia de Alimentos, Campinas, v. 17, n. 1, p. 64-69, 1997.

VIEIRA, F. C. Efeito do tratamento com calor e baixa umidade sobre características físicas e funcionais dos amidos de mandioquinha-salsa (Arracacia xanthorhiza), de batata doce (Ipomoea batatas) e de gengibre (Zingiber officinale). 2004. 103 p. Dissertação (Mestrado em Ciência e Tecnologia de Alimentos)-Escola Superior de Agricultura Luiz de Queiroz, Universidade de São Paulo, Piracicaba, 2004. 